\title{
ATMOSPHERIC SCATTERING EFFECTS ON GROUND-BASED MEASUREMENTS OF THERMOSPHERIC WINDS
}

\author{
V. J. ABREU, G. A. SCHMITT, P. B. HAYS, J. W. MERIWETHER, Jr. and C. A. TEPLEY \\ Space Physics Research Laboratory, University of Michigan, Ann Arbor, MI 48109, U.S.A. \\ and \\ L. L. COGGER \\ Department of Physics, University of Calgary, Calgary, Alberta, Canada
}

(Received 30 August 1982)

\begin{abstract}
Inherent in observations of thermospheric winds from the ground with the Fabry-Perot interferometer is the assumption that the measured Doppler shift is a property of the source medium viewed by the instrumental line of sight. However, ground based airglow observations in regions of weak airglow emission near large intensity gradients may be contaminated by scattered light. Light from areas where the emission is strong can be scattered by the lower atmosphere into the field of view of the observations. Thermospheric winds deduced from the observed Doppler shifts will then show apparent convergence or divergence with respect to the site of observation. Examples of this effect are found in observations by the Michigan Airglow Observatory station located near the auroral zone at Calgary, Alberta. Simulation calculations based upon an experimental model for a significant scattering atmosphere also showed results with either convergence or divergence in the apparent neutral wind field observed by the station.
\end{abstract}

\section{INTRODUCTION}

The measurement of the Doppler shift of atomic lines with a Fabry-Perot interferometer is a common technique presently used to study the dynamics of the Earth's upper atmosphere from ground and space (Armstrong, 1969; Hays and Roble, 1971 ; Hernandez and Roble, 1976a, b; Hernandez and Roble, 1978; Hernandez and Roble, 1979; Hays et al., 1979; Hernandez, 1980; Burnside et al., 1981; Hays et al., 1981; Hays, 1982). The interpretation of these measurements has always assumed that the source of the observed brightness consists primarily of nonscattered light. Recently, however, observations made at the Michigan Airglow Observatory (MAO) sites at Calgary $(51.08 \mathrm{~N}, 114.1 \mathrm{~W})$ and at Ann Arbor $(42.22 \mathrm{~N}$, $83.78 \mathrm{~W}$ ) have led us to re-examine this assumption. The concern arose as we found situations similar to the one displayed in Fig. 1. Here we show the wind field deduced from measurements of the $6300 \AA \mathrm{O}\left({ }^{1} \mathrm{D}\right)$ emission made at the Calgary station as a function of latitude and time. The details concerning the instrumental aspects of the automated Calgary observatory and the derivation of the wind field presented in Fig. 1 are given by Meriwether et al. (1982) and Tepley et al. (1983), respectively.

Figure 1 shows a diverging wind field pattern before midnight, while in the early hours of the morning a converging field prevails over the Calgary station. The intensities from which these winds were derived are as high as $1 \mathrm{kR}$ in the region of divergence and several hundred Rayleighs after midnight when the converging pattern is observed. The intensities measured lead to small error bars and high confidence in the derived wind vectors. From these observations one could infer that the observatory was at the boundary between two flow patterns of different origin. There is, however, an alternative explanation. In this paper we will show how a converging or diverging wind pattern can be obtained in the presence of strong intensity gradients (such as those that may be found in auroral activity) and a scattering atmosphere.

In the presence of a uniform emission layer, as depicted in Fig. 2a, a uniform wind blowing from South to North would be observed as a red shift or as a blue shift in the emission line depending on whether the direction is to the North or South of the station, respectively. The combination of intensity gradients and a scattering atmosphere makes it possible that the primary source of the observed brightness will be scattered light derived from the scattering by aerosols residing in the lower atmosphere near the station. The contribution by Rayleigh scattering for the wavelength of $6300 \AA$ is insigificant. Figure $2 \mathrm{~b}$ shows an example of 


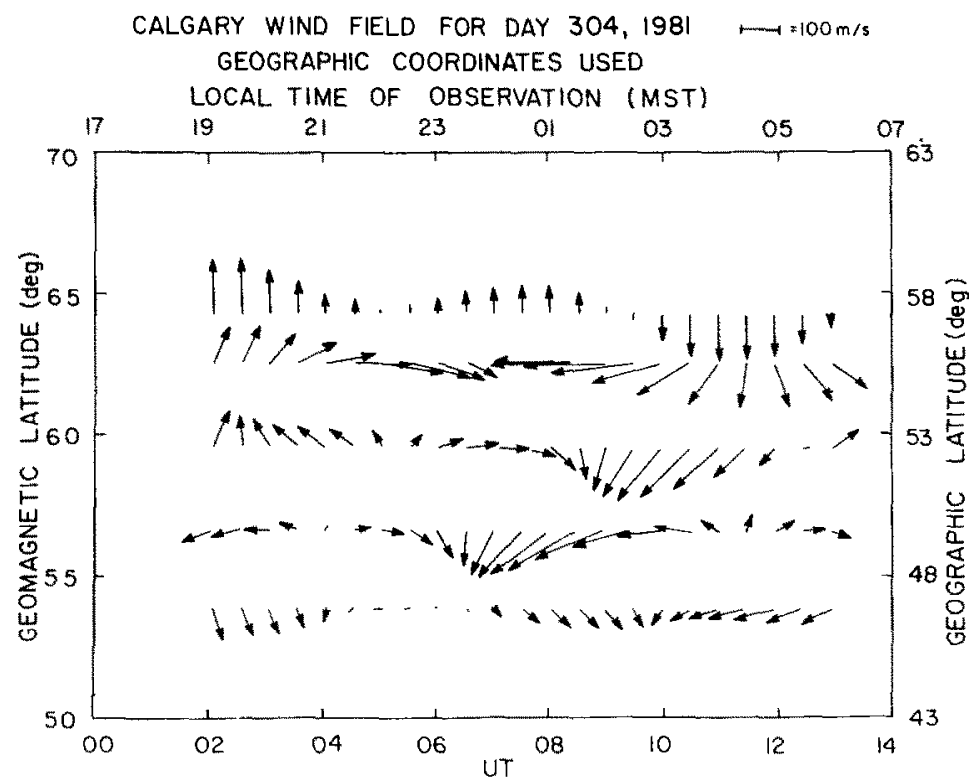

Fig. 1. WIND FIELD DEDUCED FROM MEASUREMENTS OF THE $6300 \AA$ O(1D) EMISSION MADE AT THE MICHIGAN Airglow Observatory stte at Calgary, Alberta.

this situation in which the wind is blowing from South to North. When observing to the North since the intensity of the scattered light is small compared to the direct emission, a red shift would be observed implying the presence of a northward wind. In the absence of direct emission a measurement towards the South would see only the light scattered from atmospheric aerosols and consequently, a red shift in the emission. This red shift would be interpreted as a southward wind. The set of measurements would therefore imply a situation of diverging meridional winds with respect to the station. Similarly, a converging wind pattern could be produced by reversing the actual direction of the wind or displacing the emission layer to the south of the station. We present the results of numerical simulations of this phenomenon that were carried out to assess its importance.

\section{THEORETICAL DEVELOPMENT}

The brightness, $B_{m}(\lambda, \theta)$, measured when observing an emission layer in the presence of a scattering

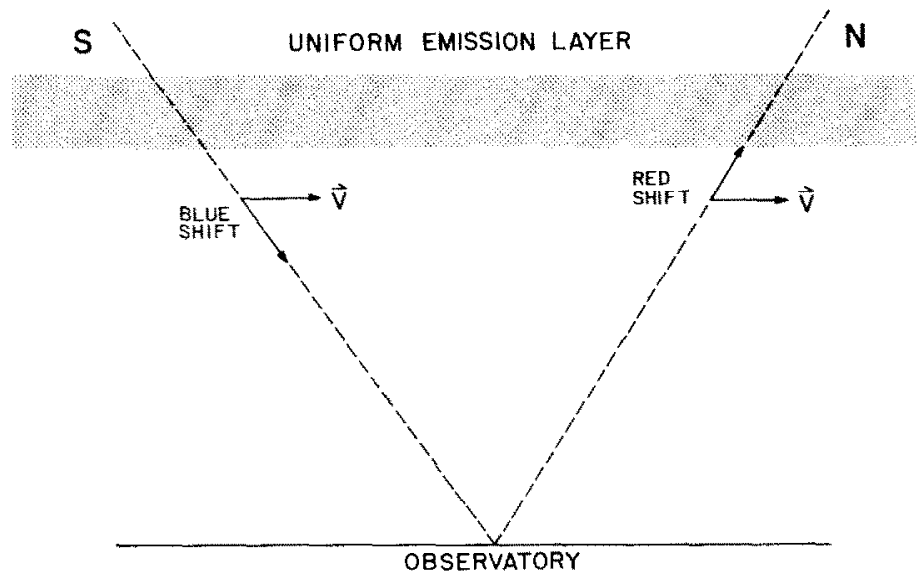

FiG. 2a. SCHEMATIC SHOWING THAT IN THE PRESENCE OF A UNIFORM EMISSION LAYER, A MERIDIONAL WIND BLOWING FROM SOUTH (S) TO NORTH (N) WOULD BE OBSERVED AS A RED BHIFT OR A BLUE SHIFT IN THE EMISSION LINE DEPENDING ON THE DIRECTION OF OBSERVATION. 


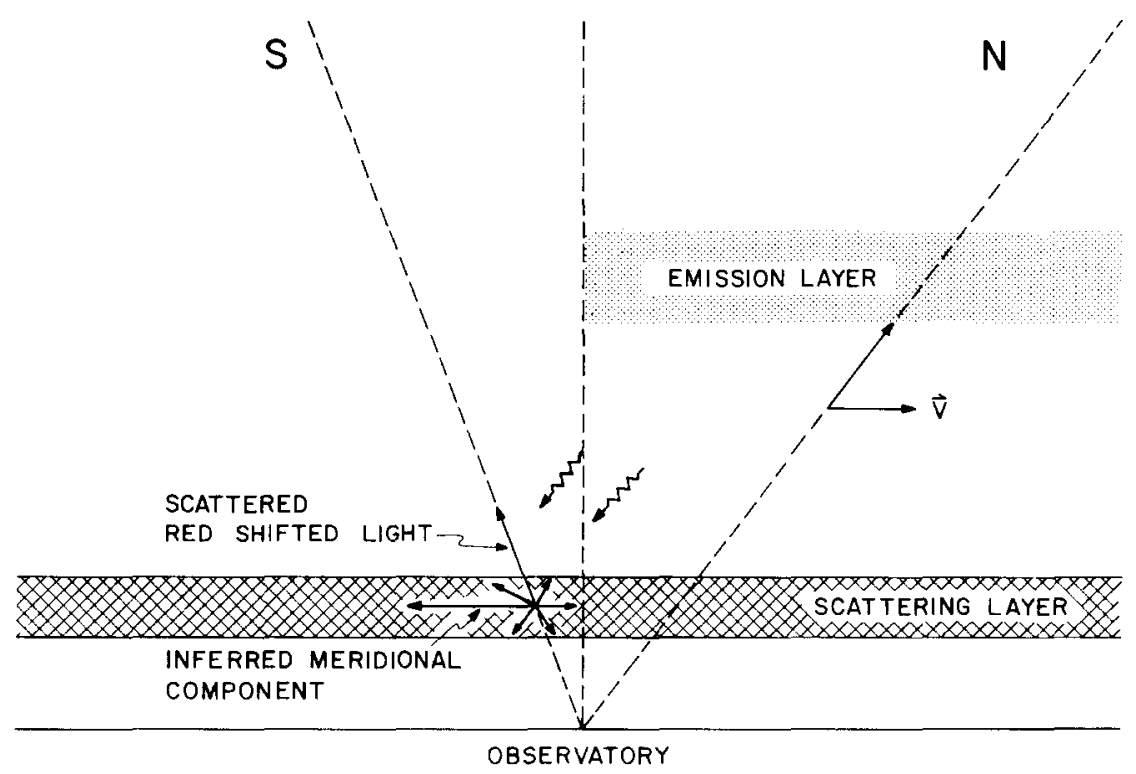

FIG. 2b. EXAMPLE SHOWING HOW IN THE PRESENCE OF INTENSITY GRADIENTS AND A SCATTERING ATMOSPHERE A DIVERGING (CONVERGING) WIND PATTERN COULD BE DEDUCED FROM THE OBSERVATIONS (SEE TEXT FOR EXPLANATION).

atmosphere consists of contributions from the direct emission and from the scattered light. Assuming that the brightness varies in only one direction, i.e., with latitude, it can be represented by

$$
B_{m}(\lambda, \theta)=\frac{B_{a}(\lambda, \theta)}{\cos \theta} \mathrm{e}^{\mathrm{r}(\lambda) \operatorname{scc} \theta}+B_{s}(\lambda, \theta)
$$

where $B_{a}(\lambda, \theta)$ is the brightness in Rayleighs of a thin emission layer at wavelength $\lambda, \tau(\lambda)$ is the optical depth of the atmosphere, $\theta$ is the angle of observation measured from the zenith, and $B_{s}(\lambda, \theta)$ is the scattering component of the observed brightness. The scattering component is given by

$$
B_{s}(\lambda, \theta)=\int_{-\pi / 2}^{\pi / 2} B_{a}\left(\lambda, \theta_{0}\right) W\left(\lambda, \alpha, \theta, \theta_{0}\right) \mathrm{d} \theta_{0}
$$

where

$W\left(\lambda, \alpha, \theta, \theta_{0}\right)=\frac{\cos \theta_{0}}{\pi} \sin \theta_{0} \int_{0}^{\pi} \sigma\left(\lambda, \alpha, 0,0_{0}, \phi_{0}\right) \mathrm{d} \phi_{0}$,

$\theta_{0}$ is the angle between an incident ray and the normal to the surface, and $\sigma\left(\lambda, \alpha, \theta, \theta_{0}, \phi_{0}\right)$ is the scattering transmission coefficient.

The scattering transmission coefficient has been obtained by an approximate solution of the radiative transfer and radiative equilibrium equations using the method of directional averaging of the radiation intensity (Sobolev, 1975). Here the atmosphere has been treated as a single scattering layer with a reflecting surface underneath characterized by an albedo, $\alpha$. The scattering coefficient is given by

$$
\begin{aligned}
& \sigma\left(\lambda, \alpha, \theta, \theta_{0}, \phi_{0}\right)= \frac{[(1-\alpha) S(\tau, \eta)+2 \alpha] S(\tau, \xi)}{4+\left(3-X_{1}\right)(1-\alpha) \tau} \\
&-\frac{1}{2}\left(\mathrm{e}^{-\tau / \eta}+\mathrm{e}^{-\tau / \xi}\right)+\left[X(\gamma)-\left(3+X_{1}\right) \eta \xi\right] \sigma_{1}(\eta, \xi),
\end{aligned}
$$

where

$$
\begin{aligned}
\eta & =\cos \theta \\
\xi & =\cos \theta_{0}, \\
S(\tau, \eta) & =1+\frac{3}{2} \eta+\left(1-\frac{3}{2} \eta\right) \mathrm{e}^{-\tau / \eta} \\
\sigma_{1}(\eta, \xi) & =\frac{1}{4}\left(\mathrm{e}^{-\tau / \eta}-\mathrm{e}^{-\tau / \xi}\right) /(\eta-\xi), \\
\cos \gamma & =\eta \xi+\left[\left(1-\eta^{2}\right)\left(1-\xi^{2}\right)\right]^{1 / 2} \cos \phi_{0}, \\
X_{1}(\lambda) & =\frac{3}{2} \int_{0}^{\pi} X(\gamma, \lambda) \cos \gamma \sin \gamma \mathrm{d} \gamma,
\end{aligned}
$$

and $X(\gamma, \lambda)$ is the Mie scattering phase function.

The wavelength shift, $\Delta \lambda$, in an emission line due to a relative velocity $\mathbf{V}$, between the emitting particle and the observing station is

$$
\Delta \lambda=\frac{\lambda_{0}|\mathbf{V}|}{c} \sin \theta,
$$

where $\lambda_{0}$ is the unshifted wavelength and $c$ is the speed of light. In the absence of scattering, the observed line shape, $G(\lambda, \theta)$, would be a gaussian whose width is a 
function of the source temperature and whose central wavelength is determined by the wind velocity according to equation 5 . The observed line shape, $H(\lambda, \theta)$, in the presence of scattering, is given by

$$
\begin{aligned}
I I(\lambda, 0)= & \frac{1}{\sqrt{\pi \beta}} \frac{B_{a}(\lambda, \theta)}{\cos \theta} \mathrm{e}^{-\tau \sec \theta} \mathrm{e}^{-\left[\lambda-\lambda^{\prime}(\theta)\right]^{2} / \beta} \\
& +\frac{1}{\sqrt{\pi \beta}} \int_{-\pi / 2}^{\pi / 2} B_{a}\left(\lambda^{\prime}, \theta_{0}\right) W\left(\lambda^{\prime}, \alpha, \theta, \theta_{0}\right) \\
& \times \mathrm{e}^{-\left[\lambda-\lambda^{\prime}\left(\theta_{0}\right)\right]^{2} / \beta} \mathrm{d} \theta_{0}
\end{aligned}
$$

where

$$
\begin{aligned}
\lambda^{\prime}(\theta) & =\lambda_{0}+\frac{|\mathbf{V}|}{c} \sin \theta, \\
\beta^{1 / 2} & =4.3 \times 10^{-7} \lambda_{0}(T / M)^{1 / 2}, \\
T & =\text { source temperature }(\mathrm{K}), \\
M & =\text { molecular weight of emitting species. }
\end{aligned}
$$

Once the line shape, $H(\lambda, \theta)$, has been calculated, the "measured" Duppler shift along the line of sight can be found by fitting this function to a gaussian. The line of sight velocity is then obtained from equation 5 . Similarly, by fitting the half-width one can determine the "measured" temperature.

\section{NUMERICAL SIMULATIONS}

Given an emission latitude profile and the optical properties of a scattering atmosphere, the equations presented in the previous section allow one to estimate the scattering contribution to the measured brightness and its effect on the meridional velocities deduced from the Doppler shift measurements. The simulations that follow have assumed the wavelength to be $6300 \AA$, an optical depth equal to 0.27 , and the measured Mie phase function presented in Table 1 (Sobolev, 1963). The optical depth value chosen was taken from the tabulation by Elterman (1968) for an elevation of sea level. We have also assumed that the source temperature is $1500 \mathrm{~K}$.

Each simulation to be presented consists of two parts: the measured brightness and the inferred velocities; both are given as a function of the zenith angle of observation $(\theta)$. The left side of Fig. 3 shows the calculated measured brightness assuming the latitudinal distribution of the source is a square function with a latitudinal extent of 4.5 degrees centered on the zenith. The height of the source function for this range of latitudes was taken to be $250 \mathrm{~km}$. The source function has been normalized to unity and is labeled "actual brightness" (solid line) in the figure. Here the ground albedo was chosen to be 0.8 , representing a snow covered ground. The calculated measured brightness (direct plus scattered emission) is represented by a dashed line.

This simulation shows that the measured brightness at angles greater than \pm 50 degrees would consist only of scattered light and represents over $20 \%$ of the actual brightness. The increase in the measured brightness with angle is due to the van Rhijn effect. The dotted line shows the results of the samecalculation with an optical depth equal to 0.153 , which corresponds to an altitude of approx. $2 \mathrm{~km}$ above sea level. In this instance the scattered light is reduced to $60 \%$ of the sea level value $(\tau=0.27)$. This simulation also assumed a northward meridional wind of $400 \mathrm{~m} \mathrm{~s}^{-1}$. The right side of Fig. 3 shows the assumed simulated velocity, the calculated measured line-of-sight velocity in the presence of scattering, and the inferred meridional velocity. Since the latitudinal variation of the source is symmetric about the zenith, the scattered light contributions to the measured brightness are equally shifted toward the red and the blue. Consequently, the actual velocity is readily recovered in this instance.

Figure 4 shows the results of a simulation similar to that in Fig. 3. Here the source function is not centered on the zenith, but has been displaced and extends 4.5 degrees to the North of zenith. Two cases are shown for albedos equal to 0.2 and 0.8 , respectively. The difference between the two cases is not significant. The brightness observed to the South in this instance consists exclusively of scattered light, which is $10-15 \%$ of the actual brightness in the North. Assuming that the actual brightness to the North was of the order of $1 \mathrm{kR}$, the observed brightness to the South would be approx. $100 \mathrm{R}$, which is considerably above the airglow intensity expected in the absence of aurora. The Doppler shift measurement would therefore be dominated by the information carried by the scattered

\begin{tabular}{|c|c|c|c|c|c|c|c|c|c|c|c|c|c|}
\hline$\gamma$ & 0 & 5 & 30 & 45 & 60 & 75 & 90 & 105 & 120 & 135 & 150 & 165 & 180 \\
\hline$X(\gamma)$ & 4.6 & 3.3 & 1.9 & 1.3 & 0.94 & 0.75 & 0.64 & 0.65 & 0.72 & 0.85 & 1.03 & 1.1 & 1.2 \\
\hline
\end{tabular}
light rather than the information present in the airglow signal. This can be seen in the right portion of the figure which shows the calculated measured wind for an actual northward wind of $400 \mathrm{~m} \mathrm{~s}^{-1}$. We note, first, that the line-of-sight velocity plot shows a vertical velocity

Table 1. Measured scattered phase function of the Earth's atmosphere 


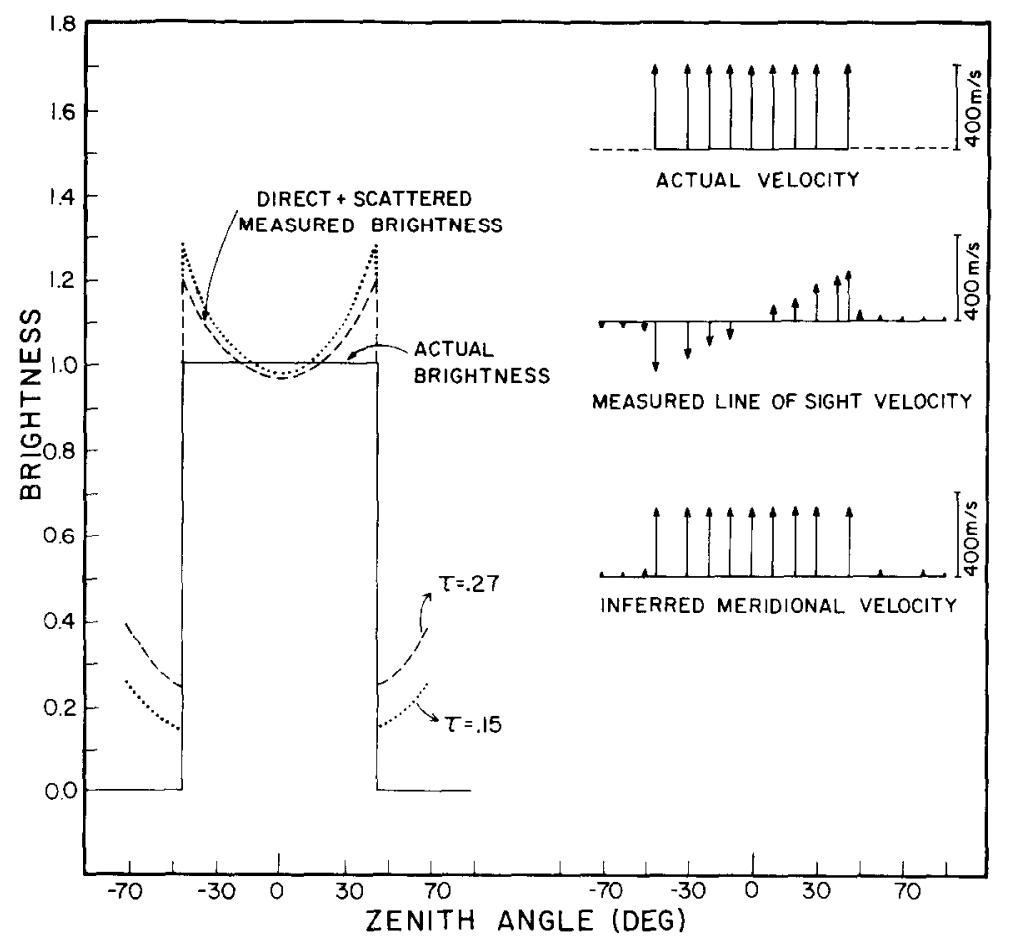

Fig. 3. CA LCULATED MEASURED BRIGHTNESSES AND VELOCITIES AS A FUNCTION OF ZENITH ANGLE OF OBSER VATION FOR A SQUARE SOURCE FUNCTION WITH A LATITUDINAL EXTENT OF 4.5 CENTERED ON THE ZENITH. SOURCE IS ASSUMED TO BE AT AN ALTITUDE OF $250 \mathrm{~km}$.

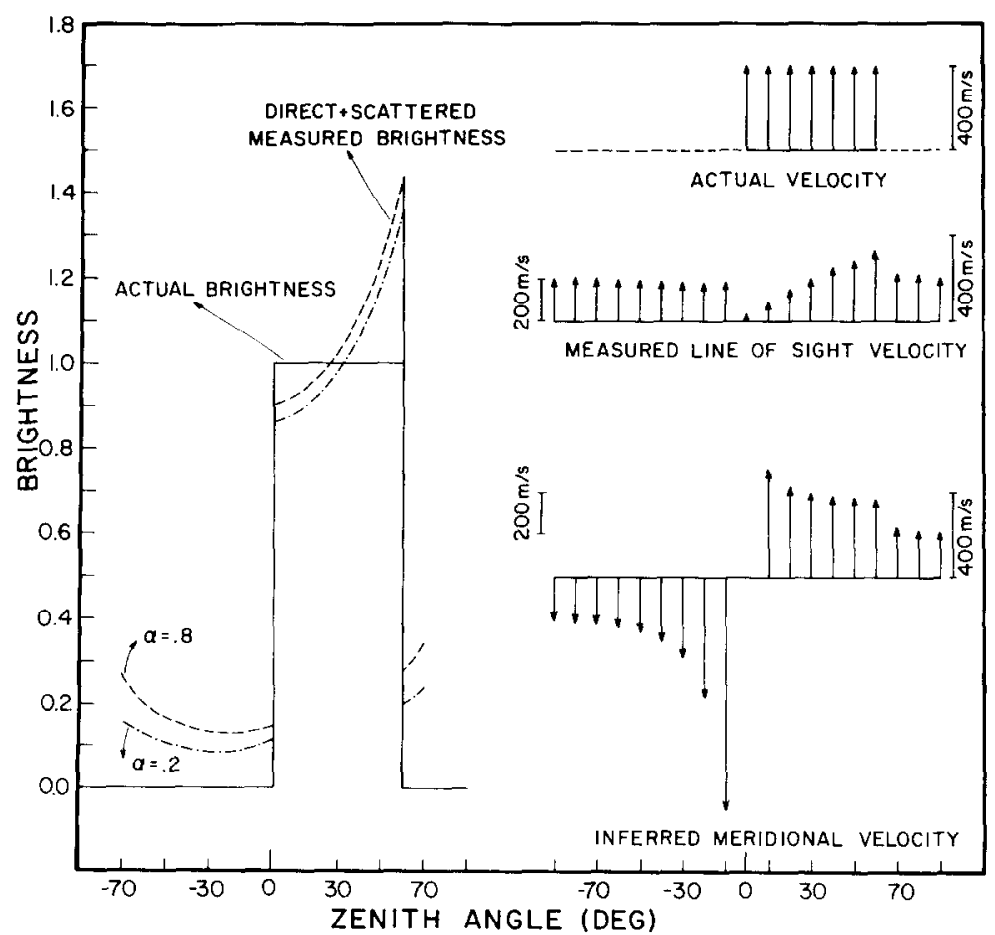

FIG. 4. CALCULATED MEASURED BRIGHTNESSES AND VELOCITIES AS A FUNCTION OF ZENITH ANGLE OF OBSERVATION FOR A SQUARE SOURCE FUNCTION EXTENDING 4.5 DEGREES FROM THE ZENITH TO THE NORTH. 
of approx. $30 \mathrm{~m} \mathrm{~s}^{-1}$ and second, that a diverging pattern emerges in the inferred meridional wind.

Further simulations have shown that as the intensity gradients are moved farther from the zenith, the importance of the scattered light contribution to the observed brightness decreases. We also found that the magnitude of the line of sight vertical velocity increases with the magnitude of the actual meridional wind.

\section{DISCUSSION}

We have shown how the presence of intensity gradients and a scattering atmosphere can be responsible for the diverging or converging patterns sometimes observed in the wind field deduced from Fabry-Perot ground-based interferometer measurements. We now present a concrete example of this phenomenon. Figure 5 shows the wind field pattern deduced from observations made at the Calgary observatory on 30/31 March 1981, a night known from visual inspection by an observer to be particularly clear. Before midnight the wind has a northward component and no convergence or divergence is observed. After midnight the meridional component of the wind in the North reverses, becoming southward. In this instance, however, a converging pattern is observed due to the lack of the apparent reversal of the southernmost measured wind vector. Figure 6 shows the measured brightness as a function of latitude (zenith angle of observation) at three different times : before, near, and after midnight. Each brightness profile has been

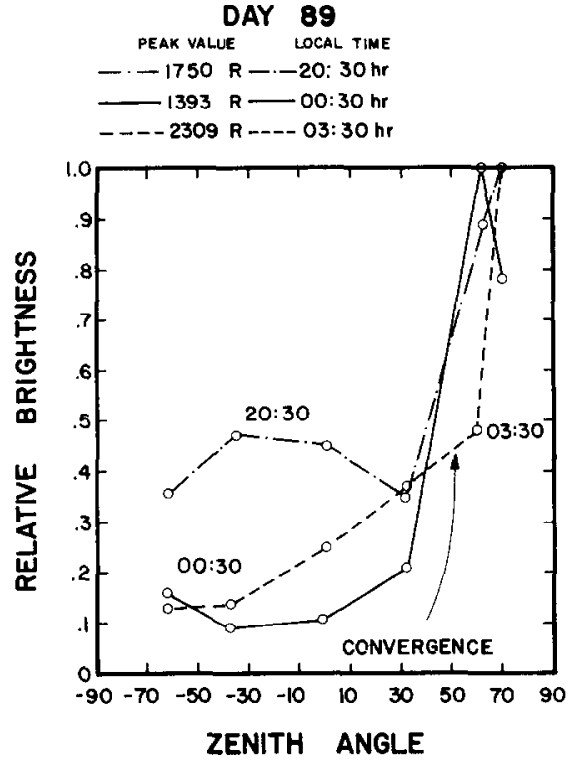

Fig. 6. MEASUREd BRIGHTNESSES NORMALIZED to PEAK VALUE AS A FUNCTION OF LATITUDE AT LOCAL TIMES CORRESPONDING TO: BEFORE, NEAR, AND AFTER MIDNIGHT, ON 30/31 MARCH 1981.

normalized to its peak value. At $20: 30 \mathrm{~h} \mathrm{~L}$.T. the direct emission dominates at all latitudes and as expected, no convergence or divergence in the wind pattern is observed. At 24:30 h most of the emission $(1.39 \mathrm{kR})$ is to the North and the resultant scattering contribution to the observed emission in the South should be small. The

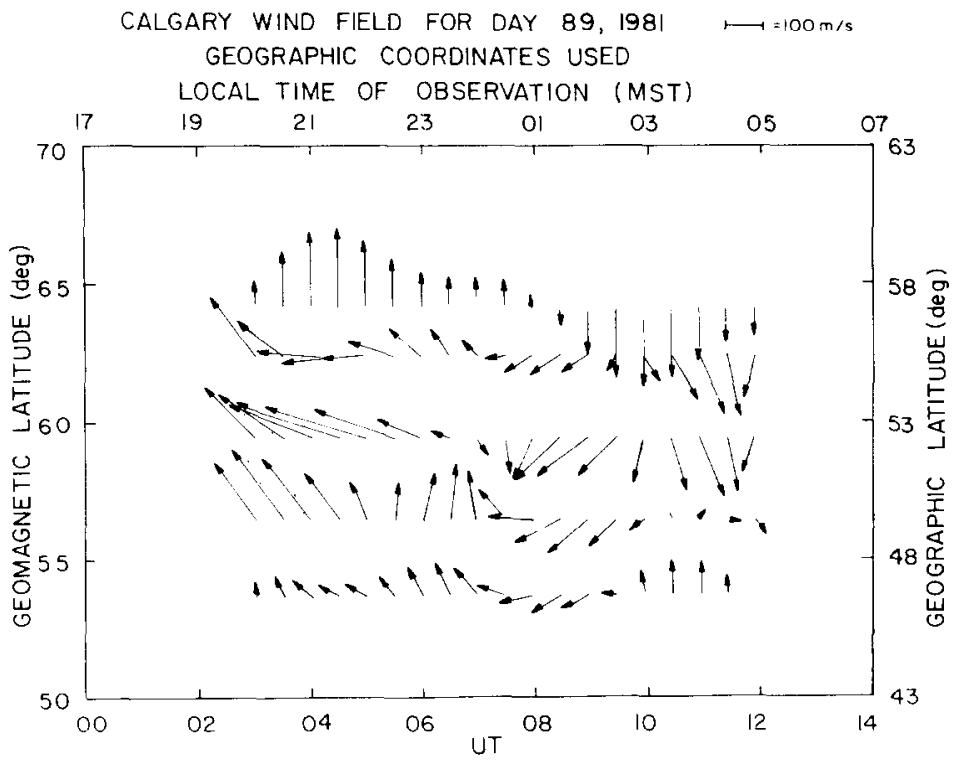

Fig. 5. WIND FIELD PATTERN DEDUCED FROM OBSERVATIONS MADE AT THE CALGARY STATION ON 30/31 MARCH, 1981. 
brightness profile at $3: 30 \mathrm{~h}$, however, has a strong gradient over the zenith and the intensity for the Southoriented observations could very well consist of mostly scattered light. This brightness distribution could produce a convergence pattern south of the Calgary station. We have simulated a situation where the source function has a strong gradient over the zenith in order to model the observations. This is shown in Fig. 7. All other parameters in the simulation are the same as those used in Section III. This simulation shows that when looking to the North of the station at the region of intense direct emission, the actual wind $\left(200 \mathrm{~m} \mathrm{~s}^{-1}\right)$ is recovered; when looking South, the scattering component becomes more significant as the angle of observation increases. This is reflected in the fact that the inferred meridional wind is about $100 \mathrm{~m} \mathrm{~s}^{-1}$ between zenith and $30 \mathrm{~S}$ and it reverses, becoming southward, beyond $30 \mathrm{~S}$. This is in agreement with the Calgary observations.

Furthermore, simultaneous observations from Fritz Peak $(39.9 \mathrm{~N}, 105.5 \mathrm{~W})$ in the northward direction show the wind blowing southward after midnight, in disagreement with the southernmost observations from the Calgary station (Hernandez, private communication). This result supports our conclusion that the convergence observed after midnight from the
Calgary station is a consequence of the presence of scattered light in the observed brightness for southward orientations.

The scattering contamination of the observed brightness also produces a broadening of the measured line profile. The deduced temperatures, however, are only enhanced by a few tens of degrees. The fact that the temperature is not very sensitive to the presence of the scattering, can be used as a clue to the true nature of the diverging or converging wind patterns, when observed. For example, the appearance of a converging pattern with a large increase in temperature to the South lends confidence to the interpretation that the observatory is at the boundary between two flow patterns of different origin. Hernandez et al. (1983) found the temperatures derived for those measurements in the South were enhanced by $200-300 \mathrm{~K}$ beyond northern values; thus, their conclusion of converging flows over their station is supported by their temperature measurements. If there is no such increase in the temperature, then the possible effects of scattered light contamination should be considered as an alternative explanation.

Another point of importance brought out by these simulations is the fact that measured vertical line-ofsight speeds may be as high as a few tens of meters per second in situations of large intensity gradients such as

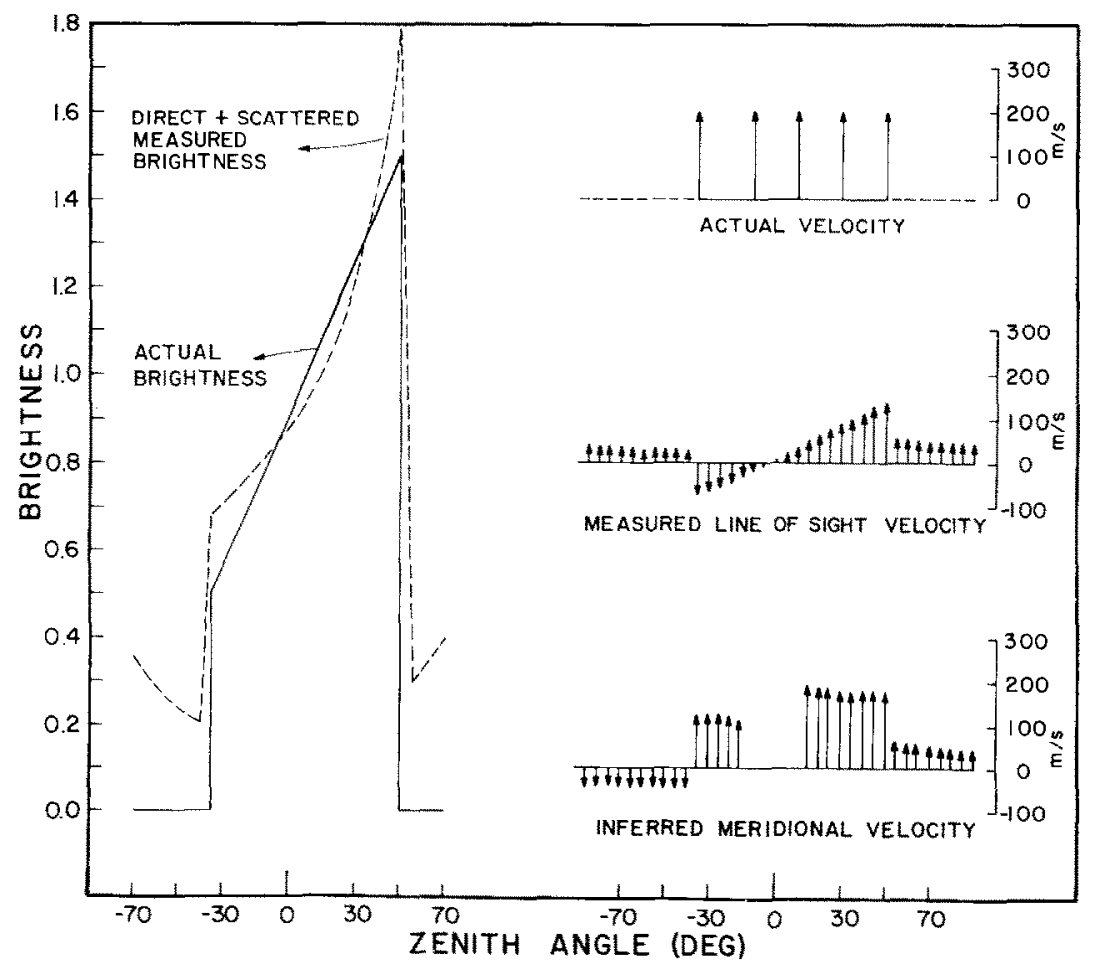

FIG. 7. CALCULATED OBSER VED BRIGHTNESSES AND VELOCITIES FOR A SOURCE FUNCTION WITH AN EMISSION GRADIENT OVER THE ZENITH. 
those often found in the aurora. Investigators who use individual zenith measurements as a reference to determine the reference for Doppler shift measurements should be aware of the possibility of scattered light contamination.

The simulations presented here have assumed that the scattering is done by aerosols, ignoring the possibility of scattering from clouds, which would enhance the degree of scattered light contamination substantially. High altitude cirrus clouds are especially important since they are not easily detectable by eye. The importance of scattering for a given wind measurement must be evaluated against the known metereological conditions and the expected aerosol distribution. The latter is very irregular in space and time. We have shown that in the absence of this information, the sky surface brightness distribution of the emission can be used to evaluate the relative contribution of scattered light to the observed brightness.

Acknowledgements - We extend our thanks to Dr Gonzalo Hernandez for various discussions. This work was supported jointly by the NSF grant ATM82-02440 and by the NASA grant NAS5-26169.

\section{REFERENCES}

Armstrong, E. B. (1969) Doppler shifts in the wavelength of the OI 6300 line in the night airglow. Planet. Space Sci. 17, 957.

Burnside, R. G., Herrero, F. A., Meriwether, J. W., Jr. and Walker, J. C. G. (1981) Optical observations of thermospheric dynamics at Arecibo. J. geophys. Res. 86, 5532.

Flterman, L. (1968) UV, visible, and IR attenuation for altitudes to $50 \mathrm{~km}$. Report AFCRL-68-0153, AFCRL, Bedford, Mass.

Hays, P. B. and Roble, R. G. (1971) Direct observations of thermospheric winds during geomagnetic storms. $J$. geophys. Res. 76, 5316.

Hays, P. B., Meriwether, J. W. and Roble, R. G. (1979) Nightline thermospheric winds at high latitudes. $J$. geophys. Res. 84, 1905.

Hays, P. B., Killeen, T. L. and Kennedy, B. C. (1981) The Fabry-Perot interferometer on Dynamics Explorer. Space Sci. Inst. 5, 395.

Hays, P. B. (1982) High resolution measurements of winds from space 1. Lower atmosphere molecular absorption. Appl. Opt. 21, 1136.

Hernandez, G. and Roble, R. G. (1976a) Direct measurements of nighttime thermospheric winds and temperatures 1 . Seasonal variations during geomagnetic quiel periods. $J$. geophys. Res. 81, 2065.

Hernandez, G. and Roble, R. G. (1976b) Direct measurements of nighttime thermospheric winds and temperatures 2 . Geomagnetic storms. J. geophys. Res. 81, 5173.

Hernandez, G. and Roble, R. G. (1978) Observations of largescale thermospheric waves during geomagnetic storms. $J$. geophys. Res. 83, 5531.

Hernandez, G. and Roble, R. G. (1979) Thermospheric dynamics investigations with very high resolution spectrometers. Appl. Opt. 18, 3376.

Hernandez, G. (1980) Measurement of thermospheric temperatures and winds by remote Fabry-Perot spectrometry, in Optical Engineering. Society of Photo-Optical Instrumentation Engineers.

Hernandez, G., Roble, R. G., Ridley, E. C. and Allen, J. H. (1982) Thermospheric response during magnetic storms during solar cycle maximum over Fritz Peak, Colorado. $J$. geophys. Res. 87, 9181.

Meriwether, J. W., Jr., Tepley, C. A., Hays, P. B. and Cogger, L. L. (1982) Remote ground-based observations of terrestrial airglow emissions and thermospheric dynamics from Calgary, Alberta. J. opt. Eng. (in press).

Tepley, C. A., Meriwether, J. W., Jr., Hays, P. B., Hernandez, G. and Cogger, L. L. (1983) Spatial distribution of the thermospheric neutral wind field (in preparation).

Sobolev, V. V. (1963) Treatise on Radiative Transfer. van Nostrand, Princeton, N.J.

Sobolev, V. V. (1975) Scattering in Planetary Atmospheres. Pergamon Press, Oxford. 